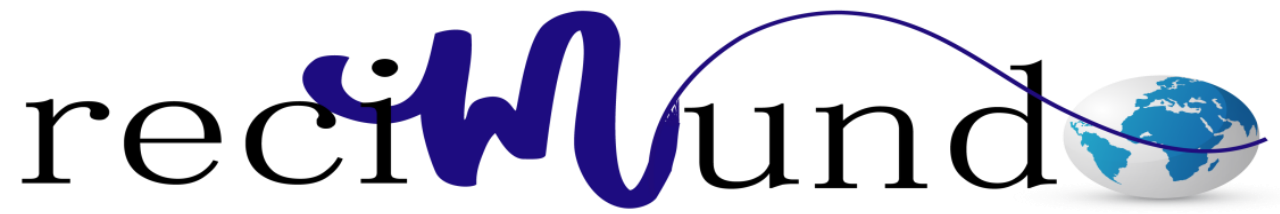

Revista Científica Mundo de la Investigación y el Conocimiento

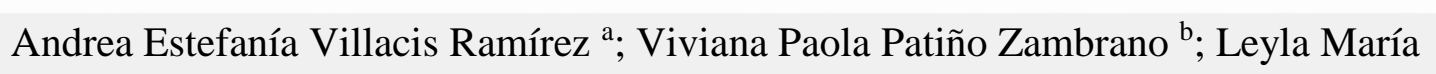
Cedeño Ávila ${ }^{\text {c; }}$ Adriana María Zambrano Solórzano ${ }^{\mathrm{d}}$

Observación de los principios Bioéticos y su aplicación en la investigación

Revista Científica Mundo de la Investigación y el Conocimiento. Vol. 2 núm., especial, mayo, ISSN: 2588-073X, 2018, pp.157-176

DOI: 10.26820/recimundo/2.esp.2018.157-176

Editorial Saberes del Conocimiento

Recibido: 15/12/2017

Aceptado: $27 / 02 / 2018$

a. Magister en Administración de Empresas con Mención en Logística y Transporte de la Universidad de Guayaquil; Ingeniera en Gestión Empresarial Internacional de la Universidad Católica de Santiago de Guayaquil; andrea.villacis.ramirez@gmail.com

b. Docente de la Universidad de Guayaquil; vivianapaopat@hotmail.com - viviana.patinoz@ug.edu.ec

c. Hospital General de Chone; Cargo: Medico General Asistencial; josearmandojaca@gmail.com

d. Médico Cirujano de la Universidad Laica Eloy Alfaro de Manabí; adrizs2009@ @otmail.com 


\section{Observación de los principios Bioéticos y su aplicación en la investigación}

Vol. 2, núm. Esp., (2018)

Andrea Estefanía Villacis Ramírez; Viviana Paola Patiño Zambrano; Leyla María Cedeño Ávila; Adriana María Zambrano Solórzano

\section{RESUMEN}

La investigación en la medicina conlleva a generar factores contractuales, del cual el profesional de la salud en cuanto a la investigación científica, en muchos casos es ético y profesional, de lo cual llega a cumplir con todos los parámetros legales del Estado Ecuatoriano, caso diferente de aquellos que inician en la investigación científica y sobre estimulan los estudios ya realizados y los hacen parte de su estudio, generando anomalías y falta de respeto ante el desarrollo intelectual de otro profesional. El objetivo de la investigación: Determinar cuáles son los valores de ética profesional en la investigación científica. Métodos; deductivo-analítico-lógico, la metodología: es el análisis deductivo-analítico-lógico. Los resultados de este análisis conllevan al entendimiento de la ética profesional adherida a la investigación científica, la inmoralidad y los aspectos educativos en el área de salud del ser vivo. Discusión se genera un análisis bajo la teoría y otros estudios ante la bioética y su aplicación a la investigación científica, y se concluye en que la conducta profesional en el área de salud, está siendo afectada por la falta de ética y moralidad ante la investigación científica por el autor.

Palabras Clave: Bioética, investigación científica, salud, legislación. 


\title{
Observación de los principios Bioéticos y su aplicación en la investigación
}

Vol. 2, núm. Esp., (2018)

Andrea Estefanía Villacis Ramírez; Viviana Paola Patiño Zambrano; Leyla María Cedeño Ávila;

Adriana María Zambrano Solórzano

\begin{abstract}
Research in medicine leads to generate contractual factors, of which the health professional in scientific research, in many cases is ethical and professional, which is in compliance with all legal parameters of the Ecuadorian State, if different of those who initiate scientific research and stimulate the studies already carried out and make them part of their study, generating anomalies and lack of respect for the intellectual development of another professional. The aim of the research: to determine the values of professional ethics in scientific research. Methods; Deductive-analytical-logical, methodology: is the deductive-analytical-logical analysis. The results of this analysis lead to an understanding of the professional ethics adhered to scientific research, immorality and educational aspects in the area of health of the living being. Discussion is generated an analysis under the theory and other studies before bioethics and its application to scientific research, and it is concluded that the professional conduct in the area of health, is being affected by the lack of ethics and morality to scientific research by the author.
\end{abstract}

Key words: Bioethics, scientific research. 


\section{Observación de los principios Bioéticos y su aplicación en la investigación}

Vol. 2, núm. Esp., (2018)

Andrea Estefanía Villacis Ramírez; Viviana Paola Patiño Zambrano; Leyla María Cedeño Ávila; Adriana María Zambrano Solórzano

\section{Introducción.}

La interesante participación de la Bioética, relacionada en su fusión ante la ética profesional y la investigación científica bajo los factores biológicos del ser humano. Casos relacionados con la investigación en el área de salud, no viene desde los tiempos actuales, esta se renueva cada década, ante la evolución del pensamiento del ser humano y la incesante participación en el área científica.

Ante este caso, los problemas morales acerca de la ciencia, mantiene falencias en la República del Ecuador, las violaciones a principios éticos, crece cada vez más a pesar de haberse generado planeación bajo la intervención universitaria, donde se pretende lograr la calidad académica que genere profesionales con capacidades y experiencias en el área de salud que sirvan para que la ciudadanía no se vea perjudicada según la patología afectada. El objetivo del estudio, Determinar cuáles son los valores de ética profesional en la investigación científica; la metodología: es el análisis deductivo-analítico-lógico. Los resultados de este análisis, conllevan al entendimiento de la ética profesional adherida a la investigación científica, la inmoralidad y los aspectos educativos en el área de salud del ser vivo. Discusión se genera un análisis bajo la teoría y otros estudios ante la bioética y su aplicación a la investigación científica, y se concluye en que la ética profesional en el área de salud, está siendo afectada por la falta de ética y moralidad ante la investigación científica por el autor.

\section{Métodos y materiales}

La investigación se generó a través de la metodología cualitativa, del cual se utilizaron las herramientas como observación y factores teórico-lógico, analítico-histórico. 


\section{Observación de los principios Bioéticos y su aplicación en la investigación}

Vol. 2, núm. Esp., (2018)

Andrea Estefanía Villacis Ramírez; Viviana Paola Patiño Zambrano; Leyla María Cedeño Ávila;

Adriana María Zambrano Solórzano

Entre los métodos y materiales se generan los siguientes:

Deductivo. - Se analiza el contenido en base a la historia de la ética de investigación de la biología humana ${ }^{1}$.

Analítico. - Conlleva a que la investigadora analice de forma sistemática, sobre las consecuencias que tienen las malas prácticas médicas en el campo de investigación biológica ante la falta d ética profesional.

Lógico. - Las corrientes ideológicas en sus variadas posiciones, encaminan a la investigadora a verificar la teoría existente y a los sistemas de investigación científica según resultados orientativos ${ }^{1}$.

\section{Resultados.}

La ética y parámetros legales

Se considera que la ética es la disciplina científica que conlleva al estudio y genera correcciones además de brindar conductas de la bondad humana en el proceso de investigación biológico.

En base a la historia, los bioeticistas mencionan al Código de Núremberg, formulado en respuesta a las atrocidades que se cometieron en el régimen Nazi durante la segunda Guerra Mundial en base a la investigación médica. Este fue el comienzo de las regulaciones que protegen al ser vivo que fue sujeto de diferentes proyectos científicos, entre estos la creación del súper humano, en aquel tiempo se generaron 1750 víctimas con Malaria, tifus, gas, veneno, 


\section{Observación de los principios Bioéticos y su aplicación en la investigación}

Vol. 2, núm. Esp., (2018)

Andrea Estefanía Villacis Ramírez; Viviana Paola Patiño Zambrano; Leyla María Cedeño Ávila; Adriana María Zambrano Solórzano

congelación, etc., del cual ante la irracionalidad humana en aquel entonces se condenaron a 23 médicos Nazis, del cual 7 de ellos cursaron la pena de muerte ${ }^{2}$.

A pesar de estas falencias en el campo de investigación, muchos aspectos clandestinos suceden en la actualidad donde, los seres humanos son presa fácil de aquellos que requieren de un ser vivo para sus experimentos, del cual esto se relaciona a la trata de órganos, donde la investigación científica pierde su ética profesional y atenta contra la biología de un ser vivo, donde los actos delictivos se generan en base al secuestro de niños y niñas, del cual los profesionales de la salud deben intervenir y ser parte de la red delincuencial, donde primero deben quitar la vida al ser humano para luego con el cuidado y delicadeza que cada órgano requiere sacar y almacenar en contenedores fríos ${ }^{3}$.

Sumado a esto, muchos profesionales de la Salud, han perdido su ética profesional, donde la investigación sobre nuevos parámetros médicos, son gobernados por las empresas farmacéuticas, del cual, aunque mucho conocen que estas empresas generan la investigación científica, del cual se generan nuevos fármacos para controlar la serie de enfermedades que circulan en el medio ambiente y perjudica a todo el mundo.

La ambición ante la riqueza, por la venta de millones de fármacos para este control, conlleva a generar una interrogante ¿Son las grandes empresas farmacéuticas las causantes de tantas enfermedades en el mundo?

Ante esta interrogante se analiza, lo que implica los principios de la bioética médica ${ }^{4}$ La Historia de Bioética, genera el entendimiento del informe Belmont, el mismo considerado en 1972 como el punto central para poder generar la ética de investigación científica con seres 


\section{Observación de los principios Bioéticos y su aplicación en la investigación}

Vol. 2, núm. Esp., (2018)

Andrea Estefanía Villacis Ramírez; Viviana Paola Patiño Zambrano; Leyla María Cedeño Ávila;

Adriana María Zambrano Solórzano

humanos, donde se formuló los principios acordes a un marco lógico de la ética de investigaciones como de la bioética clínica ${ }^{4-5}$.

Bajos estos parámetros la conducta in correcta, podría conllevar a la bondadosa actividad de realizar el bien. En este caso, puede haber disposiciones legales que no resulten éticas, en cuyo caso no hay el deber moral de cumplirlas, y al contrario, hay obligaciones éticas que no están recogidas en las leyes, y en la mayoría de los casos, no deberían existir, en general tanto la legislación como los principios bioéticos respetan conceptualizaciones diferentes ${ }^{6}$. Acorde a estos parámetros, la legislación describe solo lo que no está permitido hacer o en otras palabras lo que está prohibido de hacer; mientras que la bioética orienta sobre lo que se debería hacer, siendo la investigación en el área de salud pública un tipo de actividad humana que, aunque no sea experimental, se realiza con seres humanos, ante esto se establece el chequeo médico físico externo, la consulta o indagación al paciente afectado sobre sus dolencias y el diagnósticos acorde a los conocimientos en medicina adquirida en años de estudios universitarios ${ }^{7}$. De esta forma, el diagnostico medico se transforma en una forma de generar investigación científica, del cual, más del $90 \%$ de los profesionales de salud, recurren a los fármacos más conocidos que podrían denotar la tranquilidad y recuperar la salud del paciente, ante este punto, los médicos indican que en caso de no haber mejoría, debería nuevamente acudir al consultorio previa cita para verificar cual es el siguiente problema y darle solución, esto sucede en casi todos los hospitales y subcentro de salud en Ecuador.

La bioética, relacionada a la legislación actual, siempre que genere un beneficio ante la dolencia del paciente, no es penalizada según el Código civil y Código Integral Penal 


\section{Observación de los principios Bioéticos y su aplicación en la investigación}

Vol. 2, núm. Esp., (2018)

Andrea Estefanía Villacis Ramírez; Viviana Paola Patiño Zambrano; Leyla María Cedeño Ávila; Adriana María Zambrano Solórzano

Ecuatoriano, que rigen en sus múltiples artículos parámetros que protejan al paciente y a su vez condena a quienes causan la perdida de la vida por mala práctica médica.

La bioética, en Ecuador, como el derecho, la medicina y otras disciplinas científicas, conlleva al entendimiento de dos campos del conocimiento. El de lo abstracto y el de lo concreto, particular o de la aplicación de dichos principios.

El análisis de un problema bioético, conlleva a la investigadora a la identificación y definición del problema que se presenta en el área de salud, acorde al marco racional y razonable para poder generar orientación de posibles cursos de acción. Tal como se ha presentado, el método tiene dos niveles de análisis ${ }^{9}$. El primero, deontológico, con el cual se contrastan las alternativas con los principios. En general los principios inmutables, en la medida que el paciente genera la concepción de la misma. En este punto los avances tecnológicos y las nuevas formas de vida propician nuevas conductas y nuevas actividades de indagación científica, dando lugar a nuevos problemas éticos.

En Ecuador, los principios bioéticos, solo han tomado parte importante de la investigación científica en la aplicación de estrategias, gracias a los nuevos sistemas tecnológicos, que están incursionando a que los profesionales de la salud en la actualidad, intenten en los últimos meses, realizar trasplantes de órganos, que hace varios años atrás solo era un sueño para muchos y para otros con recursos económicos que podrían generar vi ajes a Estados Unidos o países donde la investigación científica va con avances mayores a los de otras regiones del mundo. 


\section{Observación de los principios Bioéticos y su aplicación en la investigación}

Vol. 2, núm. Esp., (2018)

Andrea Estefanía Villacis Ramírez; Viviana Paola Patiño Zambrano; Leyla María Cedeño Ávila;

Adriana María Zambrano Solórzano

Aspectos éticos de la investigación en salud publica

La información clínica, que se mantienen en la data de cada hospital o sub centro de salud, constituye una herramienta fundamental para la investigación biomédica que conlleva a profundizar en la investigación epidemiológica, habiendo sido innumerables las aportaciones que el uso del juicio profesional realiza en la medicina clínica ${ }^{10}$.

Bajo los aspectos de investigación científica que se realiza con seres humanos, se pretende lograr el objetivo primordial en la adquisición del conocimiento generalizado, del cual conlleva a beneficiar no solo al investigador sino a la comunidad en general, pero nace un problema ante este aporte, del cual la consecución del beneficio colectivo requiere del sacrificio de varios, del cual al generar la investigación científica el paciente modelo puede generar riesgos físicos o psíquicos de la investigación ${ }^{11}$.

La investigación científica en la medicina donde se considera al humano como el principal prospecto en los tiempos actuales, por un lado, es discutible la suficiencia ética del consentimiento informado para justificar la investigación, lo que requiere el inicio de los valores o principios éticos, $\mathrm{y}$, por otro lado, , existen las diversas circunstancias en las que el consentimiento informado no es posible su obtención (pacientes incapaces de tomar la decisión o fallecidos), no es viable, o su propia obtención pone en riesgo la validez ante el estudio que el profesional de la salud lo otorga.

Bajo estos aspectos, aunque el marco legal ya es muy explícito respecto al uso de la información clínica, hay un cierto espacio para la deliberación ética y el consejo prudente. 


\section{Observación de los principios Bioéticos y su aplicación en la investigación}

Vol. 2, núm. Esp., (2018)

Andrea Estefanía Villacis Ramírez; Viviana Paola Patiño Zambrano; Leyla María Cedeño Ávila; Adriana María Zambrano Solórzano

El Código de Ética del Ministerio de Salud Pública del Ecuador, en al Art.3, indica que los valores que orienten la interrelación, decisión y practica de las y los servidores de salud, deben regirse a cumplir con respeto, inclusión, vocación de servicio, compromiso, integridad, justicia y lealtad al paciente, y, además, deben bajo los aspectos médicos garantizar los derechos individuales y colectivos de la investigación científica.

El art. 4. Indica que el profesional de Salud debe contribuir al mejoramiento de las condiciones de salud de toda la población, administrar, y rendir cuentas del uso de bienes del MSP, concienciar que al principal riqueza del MSP, es el talento humano profesional al servicio de la ciudadanía, generar el respeto a las diferencias sociales y establecer los derechos específicos, además de brindar un servicio de alta calidad técnica y humana, demostrando cumplimiento a la misión y visión de la institución y establecer la garantía ante la atención de calidad y calidez de la población en general $^{12}$.

En base a lo relacionado al tema de estudio, acorde al Art. 8. Del Código de Ética del MSP, este conlleva a que se consideren los objetos de acciones que se contrapongan a las disposiciones establecidas en dicho Código, este refleja y orienta al paciente a generar las denuncias que podrán ser ingresados a través de las herramientas virtuales y físicas disponibles para la recepción de las mismas. La dirección de Talento Humano del MSP, receptará dichas denuncias y podrá tomar las decisiones que solucione el problema frente al comité del Código de Ética, conforme a la Ley Orgánica del Servicio Público y del Código de trabajo. 


\section{Observación de los principios Bioéticos y su aplicación en la investigación}

Vol. 2, núm. Esp., (2018)

Andrea Estefanía Villacis Ramírez; Viviana Paola Patiño Zambrano; Leyla María Cedeño Ávila;

Adriana María Zambrano Solórzano

El comité de Ética, se reunirá una vez al mes, y bajo las denuncias podrá detallar al profesional de Salud las decisiones tomadas ante la falta de ética profesional ejercida por el profesional de Salud.

\section{El consentimiento informado}

En el consentimiento informado, lo más importante desde un punto de vista bioético no es su obtención sino el proceso por el que se obtiene. Este proceso ha de reunir tres caracterí $\neg$ sticas básicas: la información suficiente, su comprensión y la voluntariedad. Además, hay que tener capacidad legal para dar el consentimiento ${ }^{13}$.

Ante la investigación científica, donde se incurre al humano para poder generar el estudio por el consentimiento informado del paciente y documento que se anteponga ante cualquier proceso legal que los allegados y familiares quieran ejecutar ante el perjuicio que podría adquirir el paciente por el consentimiento médico permitido.

Acorde a los parámetros anteriores, se debe entender que los riesgos ante la vida del paciente que genera el consentimiento informado no adquieren ninguna compensación monetaria, del cual se deben minimizar los riesgos y la pérdida de tiempo y capacidad de lucro que los familiares y el propio paciente piense adquirir ${ }^{14}$.

Se debe registra, el carácter voluntario del paciente para evitar que los procesos legales incurran al apresamiento del médico investigador, del cual el paciente puede detener cualquier proceso de investigación, sin que nadie pueda detenerle, y sin que este incurra en gastos por el proceso a generarse, según parámetros de investigación. 


\section{Observación de los principios Bioéticos y su aplicación en la investigación}

Vol. 2, núm. Esp., (2018)

Andrea Estefanía Villacis Ramírez; Viviana Paola Patiño Zambrano; Leyla María Cedeño Ávila; Adriana María Zambrano Solórzano

Ante este punto, la legislación si debería condenar a la participación de cada paciente de forma voluntaria, del cual ellos al estar conscientes que daño que podrían recibir por la practica medica en su investigación, del cual podría incurrir a la no viabilidad o viabilidad del estudio.

Además de lo antes mencionado, se debe generar la información de los participantes sobre las restricciones a la base de datos existente en el área médica según su infraestructura y lugar de estación, del tipo de procesos para lograr preservar la confidencialidad, y el tipo de publicación de los resultados. Como punto final, el/la investigador/a debe generar el registro del paciente, data como: Dirección del domicilio, teléfono de casa y celular, y cualquier otra forma que se pueda contactar a el paciente voluntario o si los riesgos tomaron la vida del voluntario a los familiares del mismo ${ }^{15}$.

Ante la decisión de ser parte de la investigación científica en el área de medicina, se debe establecer aspectos sobre le tener la información completa para poder tomar la decisión del estudio, que permita la comprensión. A su vez deben evitarse tecnicismos o jergas de difícil entendimiento para el paciente ${ }^{16}$.

El sujeto debe de saber siempre que forma parte de una investigación y distinguirla de un programa de intervención o de posible mejora de la salud. También ha de entender en el caso de los estudios comparativos que es posible que no reciba el tratamiento nuevo a evaluar o que incluso puede recibir el placebo, y si la investigación no es terapéutica que no va a obtener en muchos casos beneficio tangible de la misma ${ }^{17}$.

Por último, la decisión del voluntario, debe considerar los beneficios que podría recibir la familia, (no en aspectos económicos), el investigador debe condicionar, son su autoridad o poder, 


\section{Observación de los principios Bioéticos y su aplicación en la investigación}

Vol. 2, núm. Esp., (2018)

Andrea Estefanía Villacis Ramírez; Viviana Paola Patiño Zambrano; Leyla María Cedeño Ávila;

Adriana María Zambrano Solórzano

la participación de los sujetos de estudio, podría generarse un tipo de remuneración económica, siempre y cuando el caso, genere arias pruebas y estas generen bajo resultados el aporte de inversores privados en el descubrimiento del cual el sujeto fue parte, seria antiético que la bioética generada en el paciente sea truncada por el beneficio económico a través del profesional de salud.

La protección del paciente sin capacidad para proporcionar el consentimiento

Se considera un delito, del cual el paciente voluntario, no genere el consentimiento y sin embargo pase a ser sujeto de investigación, estos deben concebir la idea del proceso jurídico ante el personal de salud y posibilidad de incurrir como culpable a la administración de la institución de salud donde se ejecutará el proceso de investigación científica ${ }^{18}$.

Bajo estos parámetros, se debe generar un tipo de protección especial, del cual consideran como principales sujetos de protección a los: niños, los deficiente mentales, pacientes que requieren asistencia sanitaria especial de emergencia, etc., este tipo de protección puede tomar variaciones, del cual en el caso de niños, no tienen la capacidad legal para otorgar el consentimiento informado, en este punto intervienen los padres siempre estos estén bajo los parámetros mentales, según raciocinio y entendimiento, a pesar de que esto suceda, el niño si tienen capacidad de pensamiento lógico, que se genera después de los 5 años de vida, él puede retirar el consentimiento y la legalidad que los padres generaron no tendría validez alguna ${ }^{17-20}$.

El tipo de consentimiento se genera por los padres y derogación o aprobación de los niños hasta los doce años de vida, la Ley permite luego de esta edad el consentimiento informado para realizar los estudios científicos que la medicina requiere, otras adaptaciones, son importantes y 


\section{Observación de los principios Bioéticos y su aplicación en la investigación}

Vol. 2, núm. Esp., (2018)

Andrea Estefanía Villacis Ramírez; Viviana Paola Patiño Zambrano; Leyla María Cedeño Ávila; Adriana María Zambrano Solórzano

aplicables a las personas con deficiencia intelectual, innata o adquirida. En estos casos, no son procedentes de ejecutar el consentimiento informado.

La participación acorde a la investigación científica a generarse en pacientes que requieren asistencia de salud especial de emergencia, genera muchos problemas éticos, del cual se pueden establecer incomprensiones ante la inhabilidad del paciente por el tipo de incapacidad que mantiene y podría llegar a concebirse la idea de que este no sea representado por un profesional en el área legal, el riesgo que este adquiere es mayor en todo momento, del cual la investigación médica, podría salvar su vida, como afectarla de forma definida, en este caso, algunas intervenciones son eficaces si se generan ante la venta terapéutica ${ }^{20}$.

Otro grupo de importancia para la investigación médica con circunstancias especiales, son todas las mujeres en etapa de gestación, no porque tengan limitaciones físicas o porque no puedan otorgar el consentimiento informado, sino porque su participación, incluye la decisión de otro ser humano que crece dentro de ella y este a pesar de no estar formado y no haber nacido también decide, se podría decir que la madre tendría el 50\% de la afirmación o denegación y el feto la otra parte, en estos casos, como regla básica, la madre que tenga una posición económica media, podrá tomar la decisión ante el feto, del cual deben presentar el mínimo riesgo para la salud de ambas para así poder determinar el concentimiento informado.

Para culminar este punto, la relación asimétrica entre el paciente y médico tratante, puede limitar la voluntariedad en el consentimiento informado, del cual se toma como decisión razonable, y donde no se permite para la investigación científica el reclutamiento de estudiantes según la gestión generada. 


\section{Observación de los principios Bioéticos y su aplicación en la investigación}

Vol. 2, núm. Esp., (2018)

Andrea Estefanía Villacis Ramírez; Viviana Paola Patiño Zambrano; Leyla María Cedeño Ávila;

Adriana María Zambrano Solórzano

\section{Privacidad, intimidad, confidencialidad y anonimato}

La privacidad o confidencialidad del caso ante la situación médica que se genere entre la relación médico-paciente, se genera en la estabilidad de la información y no divulgación de la misma, antes esta situación, los estudios epidemiológicos del bien privado habitual es la información, un caso particular es sobre el aborto por mujer que sufrió en su momento una violación, el medico puede orientarse a la ética profesional de confidencialidad o romper el vínculo del mismo y establecer la respectiva denuncia ${ }^{21}$.

Hay que entender que no solo es la información del paciente por patología presenta o enfermedad crónica que este requiere de emergencia intervención del médico tratante, también incurren en la confidencialidad, el nombre, domicilio, composición familiar, datos personales, la intimidad, religión, diagnostico de una enfermedad, casa de muerte, actividad sexual, etc. En este punto la intimidad son bienes protegidos por la Constitución aprobada en el 2008.

La confidencialidad es la característica de la información por la que su acceso o transmisión están reservados o restringidos. Puede ser requerida por el facultativo o potentado de la misma. Es además un deber ético para toda la información sobre lo íntimo. En general, la información de tipo epidemiológico se hace pública preservando el nombre o identidad de los sujetos concernidos, es decir de forma anónima. La información debe utilizarse de forma anónima ${ }^{22}$.

Algunas veces se produce un conflicto aparente entre la obligación de preservar la privacidad y la intimidad, y el interés de acceder a dicha información, especialmente cuando se encuentra en bases de datos automatizadas, para realizar estudios epidemiológicos 


\section{Observación de los principios Bioéticos y su aplicación en la investigación}

Vol. 2, núm. Esp., (2018)

Andrea Estefanía Villacis Ramírez; Viviana Paola Patiño Zambrano; Leyla María Cedeño Ávila; Adriana María Zambrano Solórzano

potencialmente relevantes para la salud. Este conflicto tiene, en la práctica, soluciones técnicas. Algunas de estas soluciones tienen carácter legislativo, porque proporcionan a los sujetos confianza sobre la confidencialidad de la información.

\section{Revisión ética de los protocolos de investigación}

La Ley Orgánica de Salud Pública, en Ecuador, art. 207. Indica que la investigación científica en salud, así como el uso y desarrollo de la biotecnología, se realizará orientada bajo prioridades y necesidades de la población a nivel nacional, con sujeción a los principios bioéticos, con enfoque pluricultural, derechos de genero incorporando medicina tradicional y alternativa.

El Art. 208 de la misma Ley. Indica que, la investigación científica tecnológica en salud, deberá mantener una regulación controlada por la autoridad sanitaria nacional, del cual deberá coordinar con los organismos competentes, con sujeción a principios bioéticos y de derechos, previo consentimiento informado ${ }^{17,19-22}$.

También interviene la Ley de Propiedad Intelectual de la República del Ecuador, la que decreta en el Art. 1. - que el estado Reconoce, regula y garantiza la propiedad intelectual adquirida de conformidad con la Ley, en este punto intervienen las decisiones de la Comisión de la Comunidad Andina y los convenios internacional vigentes en el Ecuador ${ }^{22}$.

Es responsabilidad de todo investigador/a divulgar y publicar los resultados de las investigaciones realizadas en un ambiente de ética, pluralismo ideológico y diversidad cultural. 


\section{Observación de los principios Bioéticos y su aplicación en la investigación}

Vol. 2, núm. Esp., (2018)

Andrea Estefanía Villacis Ramírez; Viviana Paola Patiño Zambrano; Leyla María Cedeño Ávila;

Adriana María Zambrano Solórzano

También es obligación del investigador/a, devolver los resultados a las personas, grupos y comunidades participantes en la investigación.

Acorde a las normas de investigación científica bajo la ética profesional, los investigadores en el área de salud, solo serán parte de la misma, quienes por experiencia y conocimientos adquiridos entiendan de los riesgos médicos y de la compleja estructura del ser humano.

\section{Discusión.}

Acorde a la metodología presentada al inicio del documento, esta con llevo a analizar los factores deductivos, analíticos y lógicos, estos se generaron bajo la responsabilidad social, en cuanto a materia de salud, donde la investigación científica, cada vez que se pretende efectuar en humanos, no se toman las debidas precauciones para poder determinar si el paciente voluntario puede o no incursionar en el mismo, del cual debe existir un consentimiento informado para generarse el estudio correspondiente.

Los casos especiales, como en las madres embarazas, niños menores de edad previo al consentimiento de los padres estos podrían denegar dicha autorización si ya superan los cinco años de vida, las personas con problemas mentales tampoco podrían participar, del cual a pesar de haber un profesional en aspectos legales, los riesgos que este tipo de personas podrían incurrir, generaría una seria de infracciones ante el rompimiento de lo que significa bioética, y de los aspectos éticos que en la actualidad una gran parte de los profesionales de salud no son éticos en el campo profesional lo que conlleva a una serie de problemas y denuncias que al final 


\section{Observación de los principios Bioéticos y su aplicación en la investigación}

Vol. 2, núm. Esp., (2018)

Andrea Estefanía Villacis Ramírez; Viviana Paola Patiño Zambrano; Leyla María Cedeño Ávila; Adriana María Zambrano Solórzano

terminan afectándose de manera natural, sin que haya políticas y reglas que les puedan indultar ante las falencias generadas.

Se establece que la investigación científica bajo las estrategias profesionales que se establezcan deben conllevar a que la bioética en materia de salud, respete al ser vivo en su decisión y este no comprometa la salud e incluso la vida de este ciudadano/a, ante esto, la bioética en base a las ciencias de la vida, conllevan a que los aspectos lógicos y dinámicos aplicados a la ética profesional, genere el favoritismo a las ciencias de la vida y al aprovechamiento de la tecnología como tal, en base a la investigación científica aplicada al mejoramiento de la calidad humana en el mundo y en especial en el territorio ecuatoriano.

Se concluye que el estudio ante la bioética profesión al que se aplique al campo de investigación científica, conlleve a que los aspectos de naturaleza legal, correspondan ante la posible infracción en caso de que esta afecte la vida del paciente, a su vez que esta genere el consentimiento permitido para el fin correspondiente que genere la garantía de salud al apaciente y establezca el modelo científico acorde al estudio generado.

\section{Bibliografía.}

1. Aboso, E. (2014). Trata de personas (la criminalidad organizada en la explotación sexual laboral). Montevideo-Buenos Aires: Temis S.A.

2. Adela Cortina, (2012) Las fronteras de la persona; El valor de los animales, la dignidad de los hombres, Madrid: Taurus.

3. Arellano, J. Hernández, J. (2014) Ética de la Investigación Científica. Universidad Autónoma de Querétaro. 


\section{Observación de los principios Bioéticos y su aplicación en la investigación}

Vol. 2, núm. Esp., (2018)

Andrea Estefanía Villacis Ramírez; Viviana Paola Patiño Zambrano; Leyla María Cedeño Ávila;

Adriana María Zambrano Solórzano

4. Barreto D., Pacheco V.M., Dominguez F. y col, 2002. La Bioética en el Ecuador Presente y Perspectivas. En El cóndor, la serpiente y el colibrí OPS/OMS.,Editorial Monsalve Moreno. Quito, pp. 508-512

5. Bourdieu, Pierre, citado por Marafioti, Roberto (2008). Los sentidos de la comunicación. 2 edición. Buenos Aires: Biblos. 120 p.

6. Bunge, Mario (2012) Los enfermos son pacientes, no clientes. Diario La Nación, septiembre.

7. García Banderas A. y Estevez E., 2005, Bioética clínica, escenario de la praxis médica, Editorial Propumed, Quito, pp. 30-33.

8. Hernández, Rogel. (2014) Investigación Científica en los tiempos modernos. Barcelona. Prial.

9. Hinchliffe, S., et al. "Biosecurity and the topologies of infected life: from borderlines to borderlands", en: Transactions, 2012, vol. 38, pp. 531-543.

10. Iglesias, A. (2013). La trata de mujeres con fines de explotación sexual. Buenos Aires: didot.

11. Immanuel Kant, Lectures on Ethics, translated and edited by P. Heath and J.B. Schneewind, Cambridge: Cambridge University Press, 1997, p. 240. “A man... must practice kindness towards animals, for he who is cruel to animals becomes hard also in his dealings with men".

12. Joanne Zurlo, "No Animals Harmed: Toward a Paradigm Shift in Toxicity Testing," The Hastings Center report: Animal Research Ethics: Evolving Views and Practices, Volume 42, $\begin{array}{lll}\text { Issue } & \text { S1, November-December }\end{array}$ http://onlinelibrary.wiley.com/doi/10.1002/hast.2012.42.issue-1/issuetoc?globalMessage=0

13. Lagrée J., 2015 El médico, el enfermo y el filósofo, Editorial La esfera de los libros. Madrid, pp. 119- 120 


\section{Observación de los principios Bioéticos y su aplicación en la investigación}

Vol. 2, núm. Esp., (2018)

Andrea Estefanía Villacis Ramírez; Viviana Paola Patiño Zambrano; Leyla María Cedeño Ávila; Adriana María Zambrano Solórzano

14. Llano Escobar A. S. J. ,2001, Apuntes para una historia de la Bioética. En ¿Qué es Bioética?, Editorial Cenalbe, Bogotá, pp. 4-6

15. Martha C. Nussbaum, Las fronteras de la justicia, Barcelona: Paidós, 2011.

16. Ministerio de Salud Pública del Ecuador. (2014). Código de Ética Profesional del MSP. Art. 3.-4.8.

17. Monedero, P. (2017) Industria farmacéutica y ética médica. Revista Española de Anestesia y Reanimación. 54: 69-72, s.1.

18. MSP. CONASA.- CONABI.- OPS/OMS, (2009), La Bioética en la formación de recursos humanos en salud, Imprenta Activa, Quito, pp. 15-35.

19. National Research Council, National Academy of Sciences. 2011. Guide for the Care and Use of Laboratory Animals: Eighth Edition, http://www.nap.edu/openbook.php?record_id=12910\&page=25 (29-03-2013).

20. Occelli, M.; Vilar, T. y Valeiras, N. (2011). Conocimientos y actitudes de estudiantes de la ciudad de Córdoba (Argentina) en relación a la Biotecnología. Revista Electrónica de Enseñanza de las Ciencias. 10 (2): 227 - 242.

21. Peter Singer, (2009) Animal Liberation: The Definitive Classic of the Animal Movement (P.S.), New York: Harper Perennial Modern Classics; Reissue edition.

22. Tealdi J. C., (2008), Bioética de los Derechos humanos. Diccionario latinoamericano de Bioética, Publicación de UNESCO y Universidad Nacional de Colombia, Bogotá, pp. 177180 